\title{
Thermoelectric measurements of energy deposition during shock-wave consolidation of metal powders of several sizes
}

\author{
Andrew H. Mutz and Thad Vreeland, Jr. \\ W. M. Keck Laboratory of Engineering Materials, California Institute of Technology, 138-75 Pasadena, \\ California 91125
}

(Received 8 April 1992; accepted for publication 19 January 1993)

\begin{abstract}
The degree of shock energy localization within individual particles and between neighboring particles of different size was explored during shock-wave consolidation of spherical metal powders. The thermoelectric voltage generated by the passage of a shock wave through a copper powder-constantan powder interface was recorded. The sizes of the copper and constantan powders were varied between mean diameters of 40 and $98 \mu \mathrm{m}$. Shock-wave pressures of $5 \mathrm{GPa}$ were applied by flyer plate impact, and the resulting voltage versus time signals were collected with a $10 \mathrm{~ns}$ time resolution. In order to analyze the signals, a simulation of the thermocouple system was developed to account for the effects of multiple particle interactions and a slightly nonplanar copper-constantan interface. The resulting simulated voltage versus time signals are a good match for the observed signals when the size ratio of the copper and constantan particles is less than a factor of 2 , and reveal the preferential deposition of energy in smaller particles at the expense of larger particles within the size range examined. The amount of energy localized near particle surfaces was found to be a majority of all the energy, with a significant minority deposited throughout the particle bulk.
\end{abstract}

\section{INTRODUCTION}

Shock-wave consolidation of powders has been investigated over the past several decades as a means to produce novel and useful solid materials. ${ }^{1-3}$ The high-strain-rate deformation of the individual particles and interaction between particles rapidly removes voids and can bond the material into a monolithic solid. The preferential deposition of the shock-wave energy into particle interfaces results in bonding at relatively low equilibrium temperatures, and the combination of rapid densification and lower peak equilibrium temperature can preserve metastable structures. Strain energy localization during shock wave consolidation has been established by evidence of melted and frozen material near particle boundaries. ${ }^{4}$ The extent of such localization can be inferred to some extent by measuring the quantity of refrozen material. ${ }^{5}$ This type of "recovery experiment" is productive using the materials which leave readily measurable evidence of rapid solidification but reveals little of the dynamics of the process. A more revealing look into the localization of shock energy requires real time measurement of temperature changes.

There are several constraints on such measurements. First, the characteristic response time of the technique to be employed must be considerably smaller than the shockwave rise time in a porous medium. As established by Schwarz et al. ${ }^{6}$ the rise time of a shock wave, $t_{\text {rise, }}$ in a porous medium is roughly the particle diameter $D$ divided by the shock-wave velocity $V_{s}$. For a $6 \mathrm{GPa}$ shock wave in $64-\mu$ m-diam copper at an initial porosity of $40 \%$, the shock-wave velocity is $1.6 \mathrm{~km} / \mathrm{s}$, and the rise time is about 40 ns. Second, the measurement must be made without excessively perturbing the shock process itself, and in a region of the sample where the shock state is simple and well characterized. The optopyrometric measurement of temperature at a powder-window interface, for example, adds powder-window shock interactions to the problem. (It may nonetheless be a highly successful technique for measuring bulk shock effects; continuum effects are well understood, and corrections for the window impedance can be made. This technique was used to determine the effect of pressure on the melting point of iron ${ }^{7}$ ).

These criteria may be met utilizing the thermoelectric (or Seebeck) effect. Electric potential is generated by a thermal gradient in a conductor. The effect is monotonic and roughly linear over a wide temperature range in many metals. Dissimilar metals generally have different thermoelectric behavior. If two metals with different thermoelectric coefficients are joined, the temperature at the junction may be determined by measuring the electric potential (or EMF) across the ends of the wires, provided the temperature of the ends (the "reference temperature") is known. A discussion of the thermoelectric effect may be found in Solid State Physics, by Asheroft and Merman. ${ }^{8}$ Thermocouples fabricated from standardized alloy pairs such as platinum/platinum with $10 \%$ rhodium, and copper/ copper with $45 \%$ nickel (constantan) are commonly employed to measure temperature.

Embedding a conventional thermocouple in a powder would create impedance mismatches and other problems limiting the validity of the data during the rise time. If, however, the thermocouple is formed by the powder itself, this problem is avoided. More specifically, two powders of very similar shock impedance but different thermoelectric strength are layered parallel to the shock front. The EMF generated by this powder-powder thermocouple is recorded. Copper and constantan were used in these experiments. The effect of varying particle size distribution on thermoelectric output was explored using this technique.

Experiments measuring shock-generated EMF were 
first made in 1959 by Jaquesson. ${ }^{9}$ Thermocouples were imbedded into the material of interest, and EMF recorded. The difficulty in such measurements in powders lies in the extreme localization of the particle-particle interactions.

The shock impedance and thermal response of the powder differed considerably from that of the thermocouple. In addition, a host of anomalous voltages can be generated via friction between thermocouple elements, triboelectric effects, and electrical impedance problems. In spite of these difficulties, Bloomquist et al. ${ }^{10}$ successfully used a diffusion bonded thermocouple pair imbedded in a shocked media. This resulted in accurate equilibrium temperature measurements as predicted by Hugoniot calculations. Nesterenko ${ }^{11}$ first demonstrated that the shocked powders themselves could form the thermocouple. Schwarz et al. ${ }^{6}$ used copper and constantan powders to form the thermocouples, and measured the thermal EMF produced by the shock-wave passage. The signal showed an abrupt increase at the time of shock arrival at the junction, followed by a decay to an EMF value consistent with the calculated thermal shock energy. The rise time of the EMF signal was comparable to the time in which the shock wave traversed a single particle diameter. The decay rate was related to the thermal relaxation time for a particle.

\section{THERMOCOUPLE EXPERIMENT DESIGN}

Given the required thermocouple alloy compositions, we elected to procure inert-gas atomized copper and constantan powders from Ames Laboratory in Ames, Iowa, and HJE Corporation in Troy, NY, respectively. The copper powder was atomized from pure copper (CDA101), and delivered sieved into size fractions. The constantan was atomized from thermocouple stock ingot from Omega Engineering, Inc. of Stamford, CT. The constantan exhibited poor electrical conductivity, as received, and was treated in wet hydrogen at $400{ }^{\circ} \mathrm{C}$ for $2 \mathrm{~h}$ to reduce surface oxides. The constantan powders were sieved using a mechanical sieve shaker and bronze mesh screens into size fractions from 37 to $44,44-53 \mu \mathrm{m}$, etc. Scanning electron microscopy (SEM) micrographs of the powders are presented in Fig. 1.

A thermocouple target fixture and loading protocol was designed to insulate and shield the thermocouple circuit, and allow a planar thermocouple powder junction to be built. By fabricating the target out of copper, thermoelectric potential difference between the target and the copper powder were avoided. This was confirmed in the shock experiments; no EMF was measured as the shock wave traversed the copper plate-copper powder interface. The target consists of a circular plate counterbored to fit onto the end of the gas gun muzzle, ${ }^{12}$ with a short copper tube soldered onto the flat side of the disk. A quartz sleeve is slipped into the copper cylinder, providing electrical isolation within the cylinder. The copper powder, ceramic insulator disk, constantan powder, additional copper powder (to provide mechanical compressibility), and finally a porous bronze disk were progressively loaded into the target fixture, as shown in Fig. 2. The ceramic (Cotronics Corp. Brooklyn, NY) limits the thermocouple contact to the cen-
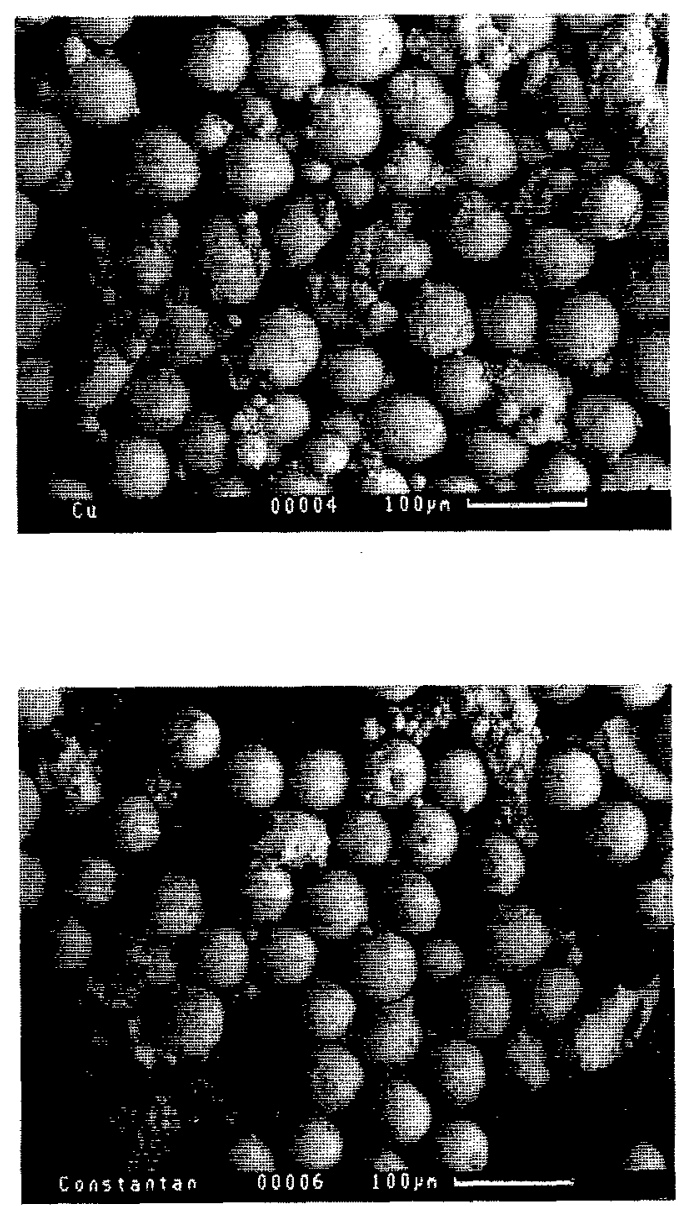

FIG. 1. Photographs of the copper and constantan powders, sieved to 63-74 $\mu \mathrm{m}$.

ter $5 \mathrm{~mm}$ of the disk. The shock wave is planar in this region of the powder; wave fronts originating at the flyer plate edge remain outside the center for the duration of the experiment. EMF is measured between the bronze plug and the copper cylinder.

The electrical circuit had to combine high input impedance and low inductance, to deliver true thermoelectric potential values with nanosecond response time. A high-

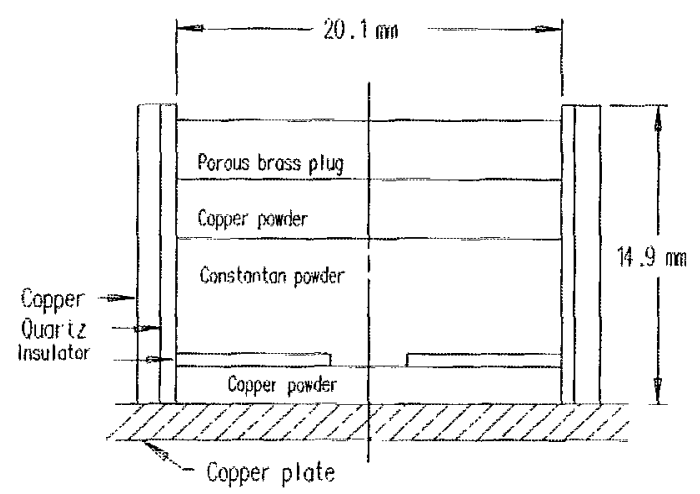

FIG. 2. Illustration of the thermocouple target, showing the powder layers. 


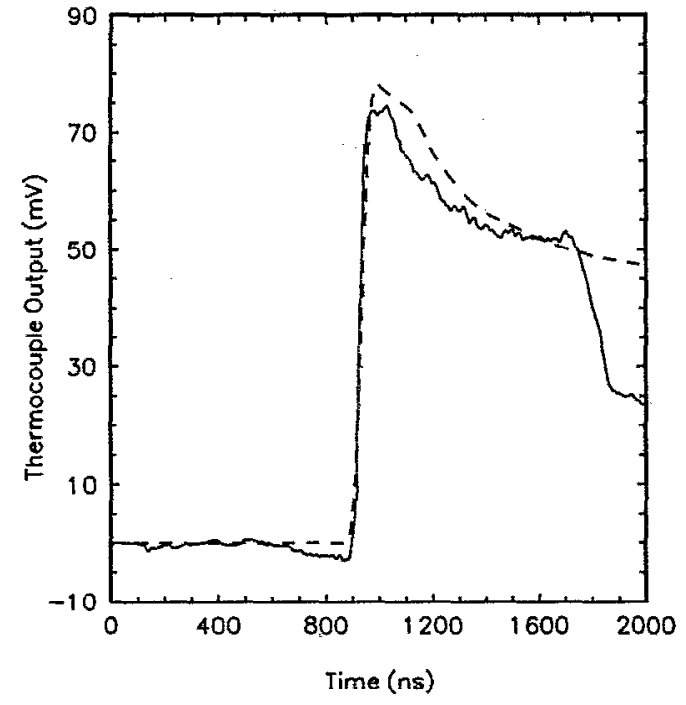

FIG. 3. Plot of thermocouple voltage vs time, from shot No. 77, and the result of the thermocouple simulation program with parameters listed in Table III. The voltage drop at $1700 \mathrm{~ns}$ corresponds to the shock wave arrival at the second copper powder layer; the constantan layer was 1.75 mm thick. (See Fig. 2).

speed op-amp video buffer follower (National Semiconductor LH0063CK) was used to construct a robust voltage follower capable of supplying the oscilloscope with an accurate signal. The signal was recorded and digitized using a CCD camera attached to the microchannel plate display screen of the oscilloscope (Tetronix 11302).

To trigger the oscilloscope at the proper time, an enameled 0.1-mm-diam copper wire was glued across the impact face of the copper target plate. The wire was kept at a $750 \mathrm{mV}$ potential, and was broken or shorted to ground by the impact of the flyer plate. The output of the wire was connected to the oscilloscope trigger with a $50 \Omega$ termination.

A 25 -mm-diam 303 SS flyer plate, mounted on a 35 mm nylon sabot, was accelerated down the evacuated barrel into the thermocouple target plate. The shock wave generated by the impact was transmitted through the copper plate and into the powder thermocouple.

\section{EXPERIMENTAL RESULTS}

The intent of these experiments was to elucidate the degree of energy segregation in a powder mixture as powders of different size are shock consolidated. If a sizable portion of the powder melts upon impact, the details of the rise time and initial temperature decay are lost in the temperature plateau created at the melting point. Therefore the velocities employed were chosen at a level generally inadequate for full interparticle bonding (though sufficient for full densification). The first experiment, with a flyer velocity of $872 \mathrm{~m} / \mathrm{s}$, resulted in a $100 \mathrm{~ns}$ voltage plateau at $75 \pm 2 \mathrm{mV}$ (see Fig. 3). The uncertainty is a result of a nonsteady zero level and 8-bit data range. This should correspond to the EMF generated by the copper-constantan couple at the melting point of copper under a pressure of 5.3 $\mathrm{GPa}$, assuming material is not heated beyond its melting point during shock consolidation. At $5.3 \mathrm{GPa}$, copper melting point is elevated to $1260^{\circ} \mathrm{C}$ based on interpolation of the results of Akella. ${ }^{13}$ Thermocouple calibration was based on data published by Bartels et al., ${ }^{14}$ and linearized to $6.5 \mu \mathrm{V} /{ }^{\circ} \mathrm{C}$; the data are not quite linear, but the interest here is in the dynamic response rather than the exact temperature value. $75 \mathrm{mV}$ corresponds to a temperature of $1175^{\circ} \mathrm{C}$, without correcting thermoelectric EMF for pressure. Since the thermoelectric signal is an average of the EMF at all points on the copper-constantan interface, a voltage plateau below that corresponding to the melt temperature can still indicate the presence of some liquid.

The thermocouple shots are summarized in Table I. A range of powder size combinations and energies was explored. The digitized traces are displayed in Fig. 3, along with the results of the thermocouple simulation.

\section{DISCUSSION AND ANALYSIS}

The analysis of this data (for elucidating the energy deposition in the powders as a function of particle size) requires analysis of the signai during the rise time and subsequent decay during the characteristic temperature relaxation time of the particles involved. Several effects interact to create the output shape. Copper and constantan spheres are in contact, thermally and electrically conducting, and mutually deforming as the shock wave consoli-

TABLE I. Powder thermocouple experiments summary. The powder sizes are as-sieved using standard mesh screens. The fiyer plate velocity was measured just prior to impact by the interruption of two light beams. The shock pressure was calculated using a Simons and Legner powder Hugoniot model, ${ }^{a}$ with parameters taken from LANL Shock Hugoniot Data. ${ }^{b}$ The equilibrium shock temperature was calculated from the shock pressure and initial porosity, using heat capacity data tabulated in the CRC Physics Handbook. ${ }^{\mathrm{c}}$ The rise time is the time required for the central $70 \%$ of the voltage change.

\begin{tabular}{rccccccc}
\hline \hline Shot & $\begin{array}{c}\mathrm{Cu} \\
\text { size } \\
(\mu \mathrm{m})\end{array}$ & $\begin{array}{c}\text { Const. } \\
\text { size } \\
(\mu \mathrm{m})\end{array}$ & $\begin{array}{c}\text { Flyer } \\
\text { vel. } \\
(\mathrm{m} / \mathrm{s})\end{array}$ & $\begin{array}{c}\text { Shock } \\
\text { press. } \\
(\mathrm{GPa})\end{array}$ & $\begin{array}{c}\text { Equil. } \\
\text { temp. } \\
\left({ }^{\circ} \mathrm{C}\right)\end{array}$ & $\begin{array}{c}\text { Maximum } \\
\text { EMF } \\
(\mathrm{mV})\end{array}$ & $\begin{array}{c}\text { Rise } \\
\text { time } \\
(\mathrm{ns})\end{array}$ \\
\hline 77 & $74-88$ & $53-106$ & 872 & 5.3 & 680 & 75 \\
80 & $90-106$ & $53-63$ & 788 & 5.0 & 550 & 59 & 50 \\
91 & $44-53$ & $74-106$ & 826 & 5.5 & 590 & 53 & 90 \\
109 & $90-106$ & $37-44$ & 726 & 4.4 & 470 & 95 \\
\hline \hline
\end{tabular}

See Ref. 16.

'See Ref. 17.

'See Ref. 18. 
dates and heats the powder particles. The thermocouple interface is composed of $25 \mathrm{~mm}^{2}$ of powder particles in contact. The interface is flat in only the roughest sense, irregularities characteristic of at least the radius of the larger particles are expected. In addition, a certain amount of mixing is possible, especially when large and small particles are combined. Some amount of average tilt is expected too, though efforts were made to control tilt to within a few degrees.

A realistic simulation of the above phenomena would require a three-dimensional finite element analysis of daunting size. More limiting is the absence of a tested model for material deformation at strain rates over $10^{7} / \mathrm{s}$. This work is limited to an examination of the thermal deposition and conduction which occurs during the shock wave passage. The heat conduction equation

$$
\frac{d T}{d t}=-k \nabla^{2} T
$$

was applied during and after the passage of an energy input front representing the shock wave. Solid density was assumed throughout. Even this problem becomes daunting when the lack of a symmetry direction, change in thermal properties, and melting of particle surfaces are included. Further simplifications render the problem tractable, although the conclusions are necessarily somewhat qualitative as a result.

The simplest dynamic representation of a particle heated preferentially on the outside consists of two thermal masses, the particle core and crust, connected by a thermal resistance giving the system the relaxation time characteristic of a sphere. The core, or inner mass, is an inner sphere concentric with the particle. The crust, or shell, is the remaining hollow sphere formed by subtracting the core from the original particle. Next, the ratio of the shell mass to the core mass, defines the relative sizes of these thermal elements. Partial melting of the spheres is considered below. If many such spheres are in contact [allow them to flatten to contact one another as in Fig. 4(a)], then the crusts will conduct to one another while the cores each conduct to only their respective crust. Note there is no net heat conduction parallel to the shock plane; simplify the picture by reducing the model to a line of particles like a string of beads along the shock direction. Connect them thermally as before. Now "blur" them (convolve them spatially by at least one particle diameter) to allow for random stacking of the many particles near the interface and the roughness of the junction itself. The "blurring" removes the periodic oscillation of temperature created by the discrete particles, but still allows a net heat flow across the interface. The "blurred" string can be simulated with relative ease.

The junction was modeled as a one-dimensional string of thermal masses conducting heat to one another and each to a single internal thernal mass [see Fig. 4(b)]. The continuum differential equations for this system (not at the junction) are

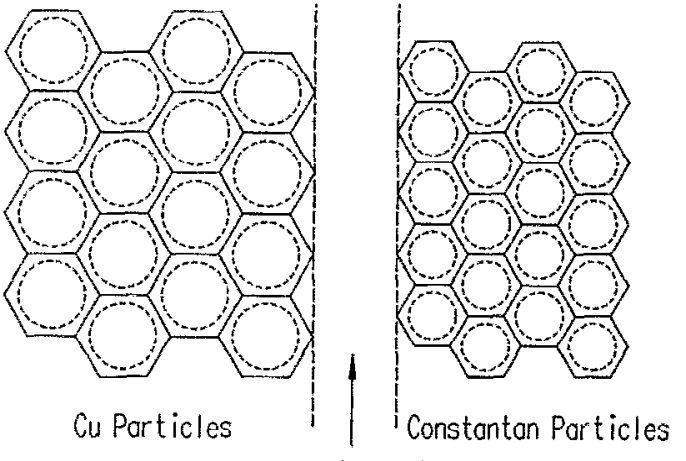

Interfoce of

Mixed Particles

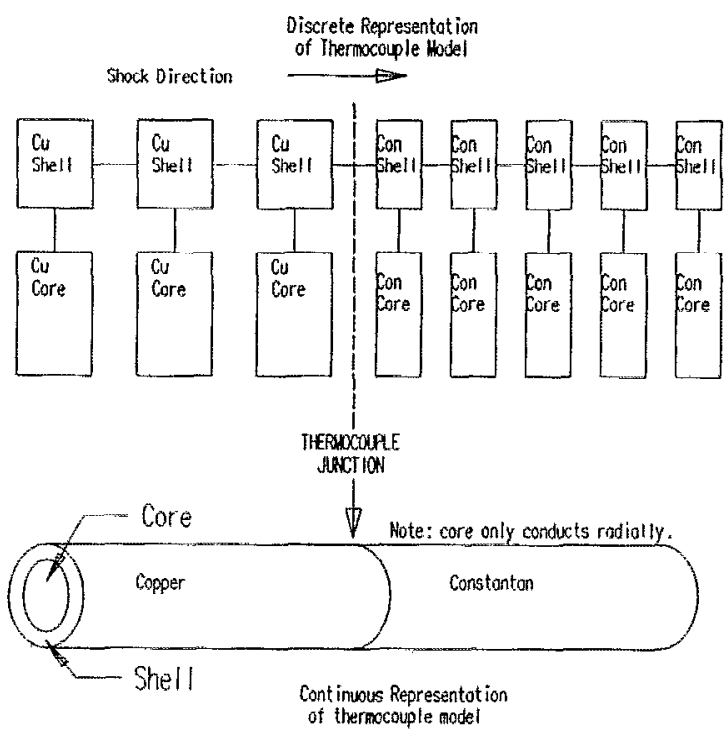

FIG. 4. Schematic representation of the thermocouple model. In (a) discrete particles of copper and constantan with surface shells (assumed to be spherical) are preferentially heated by shock deformation with respect to the cooler spherical particle cores during the shock rise time. The deformation brings the particles into intimate thermal contact. The copper-constantan interface is of finite thickness. In (b) the discrete representation, thermal masses are represented as boxes, and thermal resistances as lines. In (c) the continuous representation, the core does not conduct in the axial direction.

$$
\begin{aligned}
& \frac{d T_{0}}{d t}=-k \frac{d^{2} T_{0}}{d x^{2}}+l\left(T_{i}-T_{0}\right), \\
& \frac{d T_{i}}{d t}=m\left(T_{0}-T_{i}\right),
\end{aligned}
$$

with $T_{0}$ the particle shell temperature, $T_{i}$ the particle interior temperature, and $k, l$, and $m$ the thermal diffusion coefficients linking them. This system of equations is not analytically simple when the coefficients change abruptly and the particle shells start to melt.

The thermal properties change at the junction between copper and constantan. This may be thought of as representing a continuous copper wire butted to a constantan wire. The wire has a core which conducts only radially. The shock wave is reduced to a heat wave traveling at the shock velocity down the wire, depositing energy mainly into the outer wire, as a shock wave deposits energy mainly 
TABLE II. Copper and constantan thermodynamic data used in model. Thermal conductivity and diffusivity are from Lienhard, ${ }^{a}$ and averaged between 20 and $600^{\circ} \mathrm{C}$. Thermoelectric power is averaged from 0 to $1000{ }^{\circ} \mathrm{C}$ using data published by Bartels et al. ${ }^{\mathrm{b}}$ The melting temperature of $\mathrm{Cu}$ and melt point elevation with pressure are takcn from Akella, ${ }^{\mathrm{C}}$ and the latent heat of fusion is for $\mathrm{Cu}$ from the CRC Physics Handbook. ${ }^{\mathrm{d}}$ The melting temperature of constantan, along with the melt point elevation with pressure and latent heat of fusion, are assumed to match the properties of copper to simplify the calculations; the melting point of constan$\tan$ is actually $\sim 1320^{\circ} \mathrm{C}$ at 1 bar. $^{\text {d }}$

\begin{tabular}{|c|c|c|}
\hline Property & Copper & Constantan \\
\hline Density $\mathrm{g} / \mathrm{cm}^{3}$ & 8.9 & 8.9 \\
\hline Thermal conductivity $\mathrm{W} / \mathrm{m} \mathrm{s} \mathrm{K}$ & 385 & 35 \\
\hline Thermal diffusivity $\mathrm{cm}^{2} / \mathrm{s}$ & 1.11 & 0.101 \\
\hline Thermoelectric power $\mu \mathrm{V} / \mathrm{C}$ & \multicolumn{2}{|c|}{6.5} \\
\hline Melt temp. at $1 \mathrm{Bar}{ }^{\circ} \mathrm{C}$ & 1083 & 1083 \\
\hline$d T_{m} / d P{ }^{\circ} \mathrm{C} / \mathrm{kBar}$ & 3.36 & 3.36 \\
\hline Latent heat of fusion $\mathrm{J} / \mathrm{g}$ & 205 & 205 \\
\hline
\end{tabular}

See Ref. 19

tSee Ref. 14

'See Ref. 13.

${ }^{\mathrm{a}}$ See Ref. 18.

into the outside of a particle (see Fig. 4(b)]. In addition, the heat wave can be perturbed to deposit energy preferentially onto either of the materials near the junction. Average energy deposition is unchanged.

The final step is to allow for partial melting of the particle crust. The crust here has a mass one third of the entire particle. A simple energy sink was chosen to represent the melting transition; at each node a record of melted material is kept. If a node temperature would exceed the melting temperature, the energy is placed into this record. Upon cooling subsequent to shock-wave passage, the energy is conducted out of the melted material before any solid is cooled. The simulation stops if any particle crust is entirely melted.

As the simulation runs, the junction temperature is recorded. Following the end of the run, the resulting temperature history of the junction is convolved with a spreading function to simulate the tilt and roughness of the powder junction. The tilt of the junction is represented by a square wave convolution. Mixing is represented by a triangle wave convolution. This system was simulated and run on a PC-compatible computer. The model code, written in Microsoft QuickBasic, is listed in Ref. 15.

Averaged thermodynamic values of thermal conductivity, diffusivity, and density are listed in Table II. The melting point of copper was corrected for pressure. The melting point of constantan was assumed to match that of copper. The parameters which can be varied in the model are:

(A) the percentages of shock energy into the crust and core of the elements, $E_{\text {ext }}$ and $E_{\text {int }}$,

(B) the relative masses of the crust and core, $M_{\text {ext }}$ over $M_{\text {int }}$,

(C) the degree to which energy is preferentially deposited into the copper and constantan near the powder junction, $E_{\text {bias }}$ to $\mathrm{Cu}$, and

(D) the width of the convolution of the thermocouple output with a triangle function representing junction roughness, tilt width and mix width. These parameters allow sufficient freedom to fit almost any likely thermoelectric output. Varying all of them to achieve a best fit in each case is not the intent of this study. Rather, a reasonable determination of each parameter from the collective behavior of the experiments was attempted as follows. The results are listed in Table III.

Given the similarity of the experiments carried out, the gross character of the energy deposition was not expected to change. Thus parameters (A) and (B) were held fixed for all of the simulations. Using the duration of the voltage plateau in shot No. 77 (Fig. 3), and the shock energy required to melt material to form such a plateau as guides, parameters (A) and (B) were set at 70:30 and 1:2, respectively. If more energy is input into the particle core, material will not melt to form the voltage plateau. If the particle crust is more massive, melting is similarly suppressed.

In each shot, a somewhat different degree of thermocouple planarity existed. The mixing of copper and constantan particles was enhanced when the size difference was very large; small particles fell into the interstices between large particles. Tilting of the thermocouple junction

TABLE III. Powder thermocouple modeling parameters. The copper and constantan powder particle diameters are mean sizes. $E_{\text {tot }}$ the total shock energy, was calculated from the powder Hugoniot using the measured flyer velocity and Hugoniots from LASL Shock Hugoniot Data. ${ }^{\text {a }}$ Melt temp, the melting point of the shocked copper and constantan, was derived from the calculated shock pressure and the information in Table II. $E_{\text {ext }}$, parameter (A) in the text, is the proportion of the shock energy deposited in the outer shell of the particle in the simulation. The energy deposited in the inner core $E_{\text {int }}$ is simply (1- $\left.E_{\text {ext }}\right) \times E_{\text {tot }} \cdot M_{\text {ext }}$ over $M_{\text {int }}$ is the ratio of particle shell mass to core mass used in the simulation. These energy and mass proportions were held fixed for all the simulations, and represent a characteristic behavior of the energy deposition process. $E_{\text {bias }}$ to Cu@junc is the maximum enhancement (or reduction if negative) of energy deposition into the copper particles at the thermocouple junction, relative to the mean level. The tilt width and mix width are the half widths of the spatial convolutions used to simulate imperfections in the interface in the model. These convolutions were run over the finished thermocouple time-voltage signals which assumed a perfectly planar interface. Ebias and the convolution widths were selected for each experimental curve to optimize fit.

\begin{tabular}{|c|c|c|c|c|c|c|c|c|c|}
\hline Shot & $\begin{array}{c}\mathrm{Cu} \\
\text { diam } \\
(\mu \mathrm{m})\end{array}$ & $\begin{array}{l}\text { Con. } \\
\text { diam } \\
(\mu \mathrm{m})\end{array}$ & $\begin{array}{l}E_{\text {tot }} \\
\mathbf{J} / \mathrm{g}\end{array}$ & $\begin{array}{l}\text { Melt } \\
\text { temp } \\
\left({ }^{\circ} \mathrm{C}\right)\end{array}$ & $E_{\text {ext }}$ & $\begin{array}{l}M_{\text {ext }} \\
\text { over } \\
M_{\text {int }}\end{array}$ & $\begin{array}{c}E_{\text {bias }} \\
\text { to Cu } \\
@ \text { junc }\end{array}$ & $\begin{array}{c}\text { Tilt } \\
\text { width } \\
(\mu \mathrm{m})\end{array}$ & $\begin{array}{c}\text { Mix } \\
\text { width } \\
(\mu \mathrm{m})\end{array}$ \\
\hline 80 & 98 & 58 & 210 & 1250 & 0.7 & 0.5 & +0.25 & 37 & 25 \\
\hline 91 & 50 & 90 & 230 & 1250 & 0.7 & 0.5 & +1.0 & 50 & 12 \\
\hline 109 & 98 & 40 & 185 & 1250 & 0.7 & 0.5 & -0.5 & 25 & 25 \\
\hline
\end{tabular}

asee Ref. 17 . 

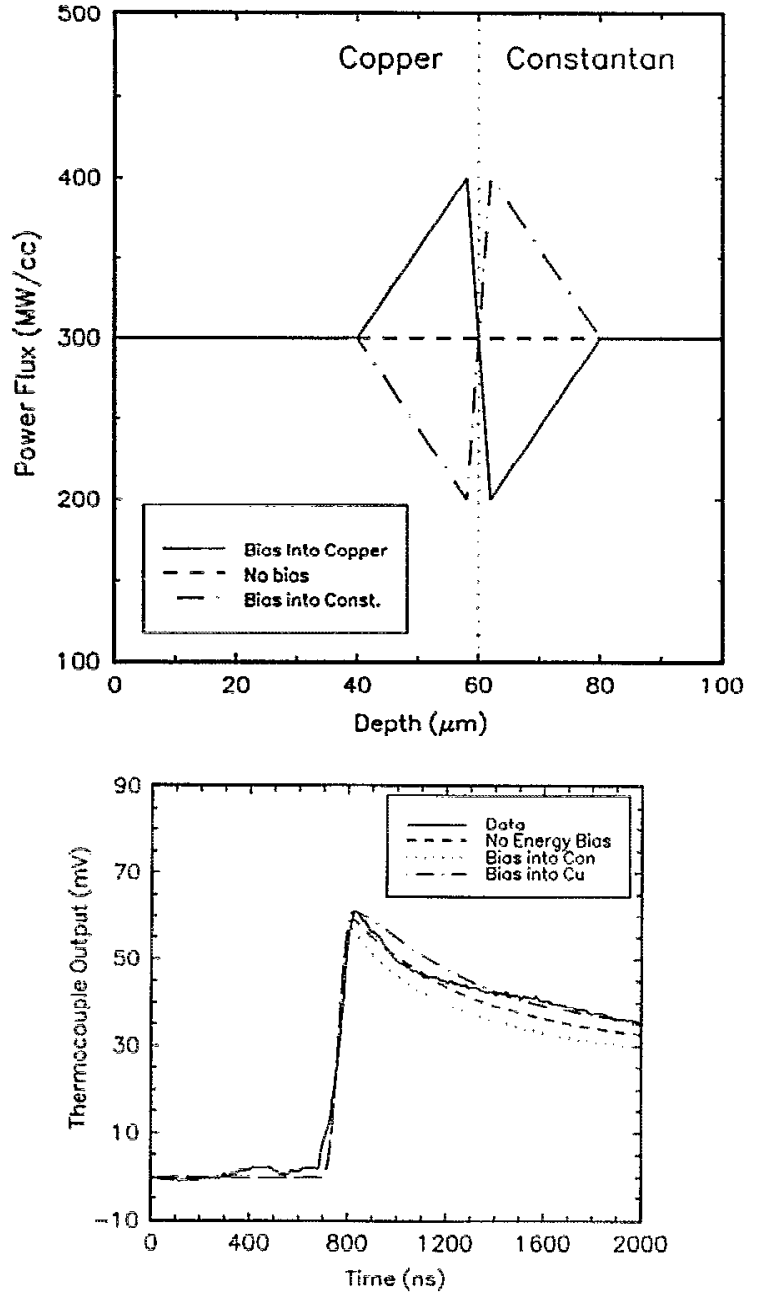

FIG. 5. (a) Plot of energy flux vs position as applied in the thermocouple model, under positive, zero, and negative energy bias into the copper. These correspond to $E_{\mathrm{ext}}=1, E_{\mathrm{ext}}=0$, and $E_{\mathrm{ext}}=-1$, respectively. (b) Plot of thermocouple voltage vs time, from shot No. 109, and the result of the thermocouple simulation program with parameters listed in Table III (labeled bias into con). In addition, the results of the simulation assuming no energy bias and energy bias into copper are shown.

relative to the shock plane varied slightly in each experiment. These effects directly influence the width and shape of the initial voltage rise of the thermocouple signal. In each experiment, parameter (D) was determined by fitting this portion of the thermocouple signal. Note that the effect of thermal relaxation during the rise time $(\sim 100 \mathrm{~ns})$ is minimal; given a thermal diffusivity of $1 \mathrm{~cm}^{2} / \mathrm{s}$, the characteristic thermal distance is $3 \mu \mathrm{m}$.

Finally, parameter (C), the shock energy distribution near the thermocouple junction, was varied as shown in Fig. 5(a). The shape of the initial voltage decay from maximum is sensitive to this variable. The energy is biased into the copper or constantan with the saw tooth profile since this conserves average energy as demanded by the Hugoniot. This is the major parameter of interest in this study of the effect of particle size distribution on energy deposition. Parameter (C) was varied to provide the best fit, especially to the initial decay slope from the voltage maximum; the accuracy of the absolute value of the maximum voltage is expected to suffer somewhat from the lack of temperaturevarying thermodynamic parameters. The model output is displayed with the data in Fig. 3. In Fig. 5(b), note the result of varying parameter (C) on the output.

As illustrated by this model, particle size strongly influences energy distribution. The fine powder, copper or constantan, is consistently heated in favor of the larger powder. In the first thermocouple experiment, shot No. 77, the wider distribution of constantan powder size (and smaller minimum size) results in more energy deposited into the constantan. The effect is not as great as in the more extreme size disparities of shot No. 109. Secondarily to particle size, hardness appears to influence energy deposition. The energy bias into the copper for best fit in shot No. $91\left(M_{\text {ext }}=1.0\right)$ is larger than the bias into the constantan in shot No. $109\left(M_{\text {ext }}=-0.5\right)$, though the ratio of sizes is similar; the copper to constantan ratio in shot No. 91 was 5:9, and in shot No. 109 was 10:4.

The simulation provides a poor prediction of system behavior when the particle sizes differ by much more than a factor of 2. Attempts to model the behavior of a shot with a size ratio of 1:3, were unsuccessful. This may be attributed to a high degree of particle intermixing at the interface, which would reduce porosity and total energy deposited near the interface.

The thermocouple simulations of shots No. 80 and No. 109 provide somewhat contradictory results; though particle sizes are similar, energy in shot No. 80 appears to be biased towards the larger copper particles $\left(M_{\text {ext }}=0.25\right)$, while in shot No. 91 energy appears to be preferentially deposited into the constantan $\left(\boldsymbol{M}_{\text {ext }}=-0.5\right)$. A certain degree of melting in shot No. $80(E=210 \mathrm{~J} / \mathrm{g})$ may be masking the detail in shot No. 80, causing an anomalously slow decay from maximum slope. A voltage plateau in shot No. $108(E=215 \mathrm{~J} / \mathrm{g})$ was observed.

\section{CONCLUSIONS}

The energy deposition process during shock-wave consolidation of spherical particles has been modeled with a one-dimensional simulation including preferential cnergy deposition at particle surfaces, preferential energy deposition into smaller particles, conduction into particle interiors, conduction between particles, and incomplete melting and freezing of particle shells.

These experiments have demonstrated (albeit qualitatively) the preferential deposition of energy in small particles in contact with larger powder particles during the shock wave consolidation process. In addition, and to a lesser degree in the hardness range tested, softer particles absorb energy preferentially to harder particles.

\footnotetext{
${ }^{1}$ N. N. Thadhani, Adv. Mater. Man. Proc. 3, 493 (1988).

${ }^{2}$ W. H. Gourdin, Mater. Sci. 30, 39 (1986).

${ }^{3}$ R. A. Graham and A. B. Sawaoka, in High Pressure Explosive Processing of Ceramics, edited by R. A. Graham and A. B. Sawaoka (Transtech, Switzerland, 1986), p. 17.

${ }^{4}$ N. N. Thadhani, A. H. Mutz, P. Kasiraj, and T. Vreeland, Jr., in Metallurgical Applications of Shock-Wave and High-Strain-Rate Phenomena, edited by I.. E. Murr, K. P. Standhammer, and M. A. Meyers (Dekker, New York, 1986), p. 247.
} 
${ }^{5}$ T. Vreeland, P. Kasiraj, A. H. Mutz, and N. N. Thadhani, in Metallurgical Applications of Shock-Wave and High-Strain-Rate Phenomena, edited by L. E. Murr, K. P. Staudhammer, and M. A. Mcyers (Dekker, New York, 1986), p. 231.

${ }^{6}$ R. B. Schwarz, P. Kasiraj, and T. Vreeland, Jr., in Metallurgical Applications of Shock-Wave and High-Strain-Rate Phenomena, edited by L. E. Murr, K. P. Staudhammer, and M. A. Meyers (Dekker, New York, 1986), p. 313.

${ }_{7}^{7}$ Q. Williams, R. Jeanloz, J. Bass, B. Svendsen, and I'. J. Ahrens, Science, 236, 4798 (1987).

${ }^{8}$ N. W. Ashcroft and N. D. Merman, Solid State Physics (Saunders, Philadelphia, 1976), p. 256.

9 J. Jaquesson, Bull. du G.A.M.A.C. IV. 4, 33 (1959).

${ }^{10}$ D. D. Bloomquist, G. E. Duvall, and J. J. Dick, J. Appl. Phys. 50, 4838 (1979).

"V. F. Nesterenko, Paper presented at the All-Union School on Explosion Physics in K rasnnyarsk, USSR (1984). English translation by the
Berkeley Scientific Trans1. Service, No. UCRL-TRANS-12054, March 1985.

${ }^{12}$ The gas gun, a $35 \mathrm{~mm}$ bore acceleration tube, is described in: A. Mutz, Ph. D. thesis, California Institute of Technology, 1991, Chap. 2.

${ }^{13}$ Akella J., J. Geophys. Chemistry 76, 4969 (1971).

${ }^{14}$ J. Bartels, P. T. Bruggencate, H. IIausen, K. II. Hellwege, K. L. Schafer, and E. Schmidt, Eds., Landolt-Bornstein: Zahlenwerte und Funktionen aus Physik, Chemie, Astronomie, Geophysik, und Technik (Springer, Berlin, 1957), p. 47.

${ }^{15}$ A. Mutz, Ph. D. thesis, California Institute of Technology, 1991, Appendix II,

${ }^{16}$ G. A. Simons and H. H. Legner, J. Appl. Phys. 53, 934 (1982).

${ }^{17}$ S. P. Marsh, ed., LASL Shock Hugoniot Data (University of California, Berkeley, 1980).

${ }^{18}$ CRC Handbook, 60th edition.

${ }^{19}$ J. H. Lienhard, A Heat Transfer Textbook (Prentice Hall, Englewood Cliffs, NJ, 1981) 